\title{
Dual EGFR and mTOR targeting in squamous cell carcinoma models, and development of early markers of efficacy
}

\author{
A Jimeno',3, P Kulesza ${ }^{1,2,3,4}$, J Wheelhouse', A Chan', X Zhang', E Kincaid², R Chen', DP Clark ${ }^{2}$, A Forastiere' \\ and $M$ Hidalgo*,I \\ 'Sidney Kimmel Comprehensive Cancer Center, The Johns Hopkins University School of Medicine, 1650 Orleans Street, Room IM89, Baltimore, MD \\ 21231-1000, USA; 'Department of Pathology, The Johns Hopkins University School of Medicine, 1650 Orleans Street, Room IM89, Baltimore, MD \\ $21231-1000$, USA
}

\begin{abstract}
The epidermal growth factor receptor (EGFR) is a validated target in squamous cell carcinoma (SCC) of the head and neck. Most patients, however, do not respond or develop resistance to this agent. Mammalian target of rapamycin (mTOR) is involved in the pathogenesis of SCC of the head and neck (SCCHN). This study aimed to determine if targeting mTOR in combination with EGFR is effective in SCC, and to develop early pharmacodynamic markers of efficacy. Two SCC cell lines, one resistant (HEP2) and one of intermediate susceptibility (Detroit 562) to EGFR inhibitors, were xenografted in vivo and treated with an mTOR inhibitor (temsirolimus), an EGFR inhibitor (erlotinib) or a combination of both. Temsirolimus exerted superior growth arrest in both cell lines than erlotinib. The combined treatment resulted in synergistic antitumor effects in the Detroit 562 cell line. Immunohistochemical assessment of pharmacodynamic effects in fine-needle aspiration (FNA) biopsies early after treatment using phospho MAPK, Phospho-P70 and Ki67 as end points demonstrated pathway abrogation in the Detroit 562 tumours treated with the combination, the only group where regressions were seen. In conclusion, an mTOR inhibitor showed antitumor activity in EGFR-resistant SCC cell lines. Marked antitumor effects were associated with dual pathway inhibition, which were detected by early FNA biopsies. British Journal of Cancer (2007) 96, 952-959. doi:I0.I038/sj.bjc.6603656 www.bjcancer.com
\end{abstract}

Published online 6 March 2007

(c) 2007 Cancer Research UK

Keywords: dual targeting; erlotinib; temsirolimus; EGFR; mTOR; squamous cell carcinoma

Squamous cell carcinoma (SCC) of the head and neck (SCCHN) is the sixth most common cancer in the developed world, affecting nearly 44000 patients and causing 11000 deaths each year in the US alone (Jemal et al, 2006). The epidermal growth factor receptor (EGFR) is a validated target in SCC of the head and neck (Bonner et al, 2006). Erlotinib (Tarceva; OSI Pharmaceuticals, Uniondale, NY, USA) and gefitinib (Iressa; Astrazeneca Inc., Willmington, DL, USA) are quinazoline derivatives that reversibly inhibit the tyrosine kinase (TK) of EGFR, showing in vitro and in vivo activity in SCCHN cell lines (Amador et al, 2004; Jimeno et $a l, 2006$ ) as well as in phase II clinical trials (Soulieres et al, 2004; Kirby et al, 2006). Tumour responses induced by these agents are, however, infrequent and transient and even sensitive patients rapidly develop secondary resistance. Thus, developing therapeutic alternatives for such patients is a necessity. The predominant mechanism underlying resistance to these agents is unknown. Failure of one single strategy to fully inhibit the pathway has been suggested and, in fact, dual targeting of the EGFR results in improved antitumor effects in preclinical models

*Correspondence: Associate Professor M Hidalgo;

E-mail: mhidalg | @jhmi.edu

${ }^{3}$ These authors contributed equally to this work.

${ }^{4}$ Current affiliation: Department of Pathology, Wallace Tumor Institute, University of Alabama at Birmingham, AL, USA.

Received II December 2006; accepted 29 January 2007; published online 6 March 2007 of SCC of the head and neck (Huang et al, 2004; Matar et al, 2004; Jimeno et al, 2005). Another hypothesis is that persistent activation of downstream pathways abrogates the effects of the agents. Thus, one attractive approach is to combine EGFR inhibitors with other signalling inhibitors targeting downstream pathways.

It has recently been shown that SCC have elevated levels of Akt activation (Amornphimoltham et al, 2004). In other preclinical models such persistent activation of Akt has been linked to resistance to EGFR inhibitors (Bianco et al, 2003). Furthermore, a substantial body of preclinical studies suggest that tumours with persistent Akt activation have heightened responses to inhibition of the mammalian target of rapamycin (mTOR) (Neshat et al, 2001; DeGraffenried et al, 2004; Gera et al, 2004). A recent study in SCC cell lines demonstrates that inhibition of mTOR induces activation of Akt and results in antitumor effects in this tumour type (Amornphimoltham et al, 2005). In this model, as well as in other disease types such as pancreas cancer (Bruns et al, 2004), the antitumor effects of mTOR inhibitors appear to be in part related to their antiangiogenic effects. In our previous work, we have determined the susceptibility of a set of SCC cell lines both in vitro and in vivo to EGFR inhibitors (Amador et al, 2004; Jimeno et al, 2006). In the current work, we tested whether MTOR inhibition with temsirolimus (Wyeth, Madison, NJ, USA) alone and in combination with erlotinib would be efficacious in those EGFR-resistant SCC in vivo models. We investigated the utility of fine-needle aspiration (FNA)-based early assessment of 
pharmacodynamic response by immunohistochemistry assessing both proximal Phospho-MAPK (pMAPK) and phospho-P70 (pP70) and distal (Ki67) markers. These early biopsies were also used to investigate changes in the expression profile of relevant genes potentially related to angiogenesis and efficacy of EGFR and mTOR inhibitors.

\section{MATERIALS AND METHODS}

\section{Drugs}

Erlotinib was provided by OSI Pharmaceuticals (Uniondale, NY, USA). Temsirolimus was provided by Wyeth (Madison, NJ, USA).

\section{Cell lines and in vitro culture conditions}

The cell lines used in this study (HEP2 and Detroit 562) were obtained from the American Type Culture Collection (Manassas, VA, USA), and are derived from cervical (HeLa contaminant) and head and neck squamous carcinomas, respectively. These cell lines were used based on our prior knowledge of their sensitivity to erlotinib as well as the existing information with mTOR inhibitors in the model. The cell lines were grown in six-well plates with RPMI media supplemented with $10 \%$ fetal bovine serum (FBS) and $1 \%$ penicillin/streptomycin (growth media (GM). When 50-60\% confluency was reached, they were serum starved overnight, after which they were exposed to (1) serum-starved media for $2 \mathrm{~h}$ and $30 \mathrm{~min},(2)$ serum-starved media for $30 \mathrm{~min}$ followed by GM for $2 \mathrm{~h}$, (3) serum-starved media plus erlotinib $(10 \mu \mathrm{M})$ for $30 \mathrm{~min}$ followed by GM plus erlotinib for $2 \mathrm{~h}$, (4) serum-starved media plus temsirolimus $(10 \mu \mathrm{M})$ for $30 \mathrm{~min}$ followed by GM plus temsirolimus for $2 \mathrm{~h}$ and (5) serum-starved media plus the combination of erlotinib $(10 \mu \mathrm{M})$ plus temsirolimus $(10 \mu \mathrm{M})$ for $30 \mathrm{~min}$ followed by GM plus erlotinib plus temsirolimus for $2 \mathrm{~h}$. After this the cells were washed twice with chilled PBS, lysis buffer was added to the plates and the cells were scraped for protein extraction. An identical experimental set was lysed directly from the tissue culture dish with RLT (Qiagen, Valencia, CA, USA) for RNA extraction.

\section{In vivo growth inhibition studies}

Six-week-old female athymic nude mice (Harlan, IN, USA) were used. $2 \times 10^{6}$ HEP2 and Detroit 562 cells were injected subcutaneously in each flank. Tumours were allowed to grow until reaching $200 \mathrm{~mm}^{3}$, at which time mice were randomized in the following four groups of treatment, with five to six mice (10 evaluable tumours) in each group: (1) control, (2) erlotinib $50 \mathrm{mg} \mathrm{kg} \mathrm{day}^{-1}$ i.p., (3) temsirolimus $20 \mathrm{mg} \mathrm{kg} \mathrm{day}^{-1}$ i.p., and (4) erlotinib plus temsirolimus at the above doses. Treatment was given for 28 days. Mice were monitored daily for signs of toxicity and were weighed three times per week. Tumour size were evaluated two times per week by caliper measurements using the following formula: tumour volume $=\left(\right.$ length $\times$ width $\left.^{2}\right) / 2$. Relative tumour growth inhibition was calculated by relative tumour growth of treated mice divided by relative tumour growth of control mice $(\mathrm{T} / \mathrm{C})$.

\section{FNA biopsies}

Fine needle aspirations (FNAs) on mice were performed according to standard cytopathologic practice under inhaled general anaesthesia (isofluorane) using $10 \mathrm{~cm}^{3}$ syringes and 25-gauge needles. During each FNA procedure, the first pass was smeared onto glass slides and used for morphologic analysis, (DiffQuik ${ }^{\mathrm{TM}}$ and Papanicoloau), the second and third passes were harvested in RLT buffer for RNA analysis, and the fourth and fifth passes in formalin for immunohistochemical purposes. Six tumours per treatment arm were biopsied at baseline and after 7 days of therapy.

\section{Western blot analysis}

Equal amounts of protein were resolved on $10 \%$ polyacrylamide gels. Gels were transferred onto nitrocellulose membranes that were incubated overnight at $4{ }^{\circ} \mathrm{C}$ with antibodies against phosphoEGFR, total EGFR, pMAPK, total MAPK, phospho-Akt, total Akt, pP70, total P70 (Cell Signaling Technology, Beverley, MA, USA) and actin (Santa Cruz Biotechnology, Santa Cruz, CA, USA). The immunoreactive proteins were detected using the enhanced chemiluminescence method (Amersham, Piscataway, NJ, USA).

\section{RNA extraction}

For RNA extraction from the FNA samples, two passes from the FNA were put in RLT lysis buffer (Mini RNeasy, Qiagen) and total RNA was extracted using the Rneasy ${ }^{\mathrm{TM}}$ Mini Kit (Qiagen). RNA was transcribed into cDNA by reverse transcription by priming with random hexamers (M-MLTV, Promega, Madison, WI, USA). The excess hexamers were removed using a column-based cleanup kit (Qiagen).

\section{Quantitative real-time RT - PCR analysis}

Samples were analysed in a blinded manner. For c-fos, egfr and HIF-1alpha determination on in vitro samples and FNAs from mice tumours, quantitative PCR was performed on an MX3000p thermal cycler (Stratagene, Lajolla, CA, USA) using SYBR green dye method to track the progress of the reactions with ROX dye added as reference. Beta-Globin DNA specific primers were used to test DNA contamination for each sample type. Three housekeeping genes (HPRT, UBC and SDHA) were run in parallel with test genes. The amount of change in the target gene between the control and experimental conditions was found by comparing the threshold cycle $(\mathrm{Ct})$ of the target gene to the geometric mean of the Ct's of the housekeeping genes. The geometric mean of the Ct's of each of the housekeeping genes, and a change in $\mathrm{Ct}$ (delta $\mathrm{Ct})$, between conditions were calculated $\left(\mathrm{dCt}_{\text {Houskeeping }}=\left(\mathrm{Ct}_{\mathrm{HPRT}}{ }^{*} \mathrm{Ct}_{\mathrm{UBC}}{ }^{*} \mathrm{Ct}_{\mathrm{SD}}\right.\right.$ HA)control $\left.-\left(\mathrm{Ct}_{\mathrm{HPRT}}{ }^{\star} \mathrm{Ct}_{\mathrm{UBC}}{ }^{\star} \mathrm{Ct}_{\mathrm{SDHA}}\right)_{\text {exp }}\right)$. The change in $\mathrm{Ct}$ for the target gene was calculated directly from $\mathrm{Ct}$ under each condition $\left(\mathrm{dCt}_{\text {target }}=\left(\mathrm{Ct}_{\text {target }}\right)_{\text {control }}-\left(\mathrm{Ct}_{\text {target }}\right)_{\text {exp }}\right)$. The efficiency of the housekeeping genes raised to their dCt divided by the efficiency of the target gene raised to its $\mathrm{dCt}$ gave a ratio between the control and experimental conditions normalised to the housekeeping genes (Ratio $=E_{\text {Target }}^{\mathrm{dCtTarget}} / E_{\mathrm{H} \text { ousekeeping }}^{\mathrm{dCtH}}$, where $E$ is the Primer efficiency and $\mathrm{Ct}$ is the Threshold cycle).

\section{Immunohistochemical analysis}

The pathology team was blinded for treatment allocation and efficacy of the samples. Five-micrometre formalin-fixed paraffin cuts were used for Ki67 staining that was performed following the manufacturer's instructions (08-0156; Invitrogen, Carlsbad, CA, USA), and scored as percentage staining nuclei. Phospho-Thr202/ Tyr204 MAPK (4376; Cell signaling Technology, Beverly, MA, USA) and phospho-p70/S6K (9206; Cell signaling ) staining was performed using citrate-steam recovery, followed by Catalyzed Signal Amplification II biotin-free tyramide kit (DAKO, Carpinteria, CA, USA). Both the intensity $(0,1+, 2+, 3+)$ and the percentage $(0-100 \%)$ of cells positive were considered. Three tumours per treatment group were analysed. For statistical analyses, an index of intensity $\times$ percentage was calculated. CD31 staining (550274; BD Pharmingen) was performed on zinc-fixed tissue (51-7538KZ; BD Pharmigen). Two methods for vessel quantification were used. First, microvessel density (MVD) was scored according to the standard methods (Weidner et al, 1991). 


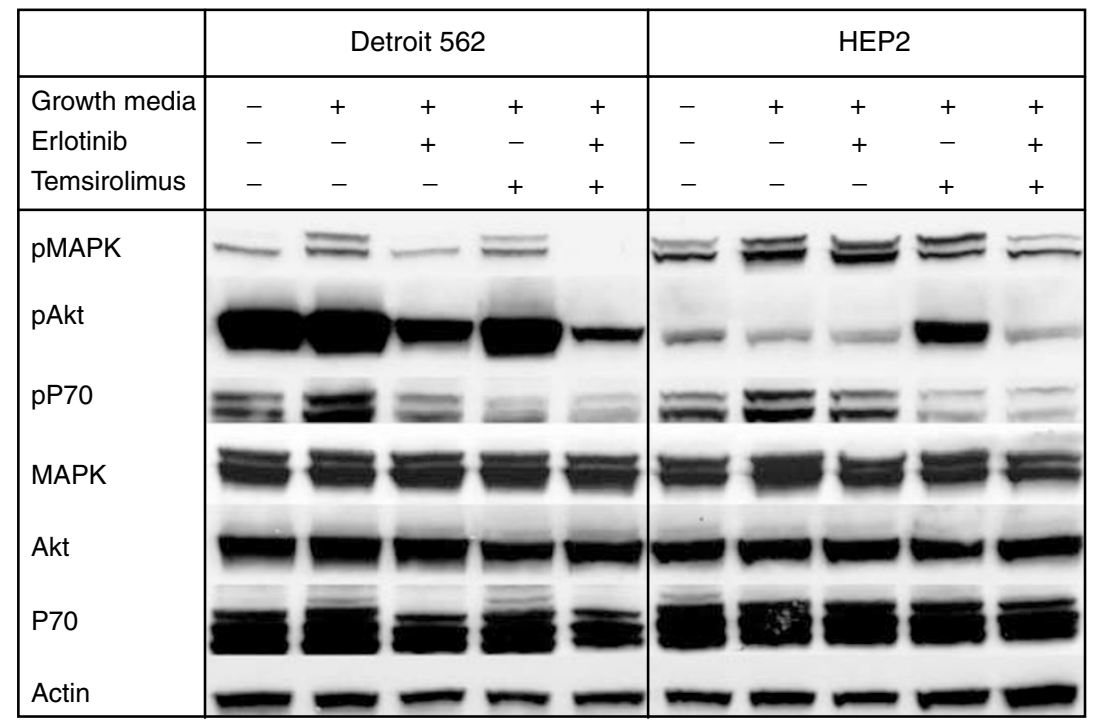

Figure I Analysis of the pathway activation profile after exposure to the study drugs. Cells were serum-starved overnight, after which they were preincubated with erlotinib $(10 \mu \mathrm{M})$, temsirolimus $(10 \mu \mathrm{M})$ and the combination of both (I0 $\mu \mathrm{M})$ followed by GM containing the same drugs. The experiment was controlled by a continuously serum starved group, and a group that received GM without preincubation with any drug. In Detroit 562 erlotinib was able to decrease PMAPK, although only the combination abrogated its activity completely. On the other hand, the effect in HEP2 was much less apparent. Phospho-Akt was significantly abrogated in Detroit 562 by erlotinib-containing arms (particularly the combination), but not by temsirolimus, indicating a more downstream level of inhibition. On the other hand, in HEP2 cells lower levels of pAkt were observed at baseline, no significant increase was seen upon exposure with GM, and no inhibition was seen after erlotinib treatment. However, a clear upregulation was observed when treated by temsirolimus alone; even more notably, this upregulation was not documented when both erlotinib and temsirolimus were combined. Inhibition of pP70 was seen in Detroit 562 and HEP2 cells after erlotinib and more markedly after temsirolimus or the combination.

Briefly, four areas per tumour were chosen and microvessel per high-power field $($ HPF; $\times 200)$ were counted. Second, we developed a novel scoring system based on quantifying mouse vessel invasion of the xenografts from the capsule. This was scored according to the following: (1) Capillaries in the pseudocapsule only, surrounding the tumour less than entire circumference. (2) Capillaries in the pseudocapsule only, surrounding the entire circumference of the tumour. (3) Capillaries in less than $50 \%$ of the deeper stroma, but not extending to the tumour centre. (4) Capillaries in more than $50 \%$ of the deeper stroma, but not extending to the tumour centre. (5): Capillaries throughout the tumour, including the centre. Six tumours per treatment group were analysed.

\section{Statistics}

The comparisons between means and proportions obtained from the biologic studies were carried out using Student's $t$-test and $\chi^{2}$ method, respectively.

\section{RESULTS}

\section{Pathway modulation analysis}

We first profiled the selected cells lines in vitro to determine the impact of the drugs in the activation of signalling pathways in this model (Figure 1). In Detroit 562, erlotinib alone inhibited MAPK activation and reduced the activation of Akt and P70. Temsirolimus predominantly inhibited P70 and induced no effects in the phosphorylation of MAPK and/or Akt. The combined treatment completely abrogated PMAPK, and significantly inhibited Akt phosphorylation. HEP2 responded similarly but the degree of inhibition was lower. Erlotinib alone had minimal effects in the signalling pathways assessed; temsirolimus, however, induced Akt activation and blocked P70, and the combination exerted increased inhibition of pMAPK with a clear abrogation of temsirolimusinduced Akt activation in this cell line.

\section{In vivo growth inhibition analysis}

The in vivo data are summarised in Figure 2. In HEP2, considering the growth of the control group from treatment commencement to the end of the experiment after 21 days as a $100 \%$ growth, erlotinib alone did not delay growth significantly, reaching $56 \%$ of the control volume by 21 days (Figure 2A). Temsirolimus slowed growth to $32 \%$, and the combination to $22 \%$ of the control group. This represents an average tumour size of 230 and $191 \%$ compared to the initiation of the experiment for the latter two treatment groups, respectively.

In Detroit 562, erlotinib alone delayed growth to $34 \%$ of the control volume by 21 days (Figure $2 \mathrm{~A}$ ), which corresponds to a $198 \%$ growth from the start of the trial. Temsirolimus slowed growth to $13 \%$, and the combination achieved a $-18 \%$ of the control group, representing a final tumour size of $136 \%$ for temsirolimus and a decrease to $49 \%$ of the initial volume in the combination arm (the only where actual tumour regressions were seen).

\section{Tumour immunohistochemical analysis}

In each xenograft model, 16 tumours (four per treatment arm) were followed by FNA at baseline and on day 7 of treatment for immunohistochemical analysis. The results are shown in Figure 3. In the comparison of the baseline FNA samples, the proliferation index Ki67 was higher in HEP2 than in Detroit 562 tumours (91 vs $74 \%, P=0.041$ ), whereas the activation status index (intensity $\times$ percentage) of MAPK was higher in Detroit 562 tumours (60 vs $119, P=0.018)$. This was related to a higher percentage of cells staining positive ( $24 v s 49 \%, P=0.038$ ), as the intensity in both groups was similar. Phospho-P70 staining index was high in both 

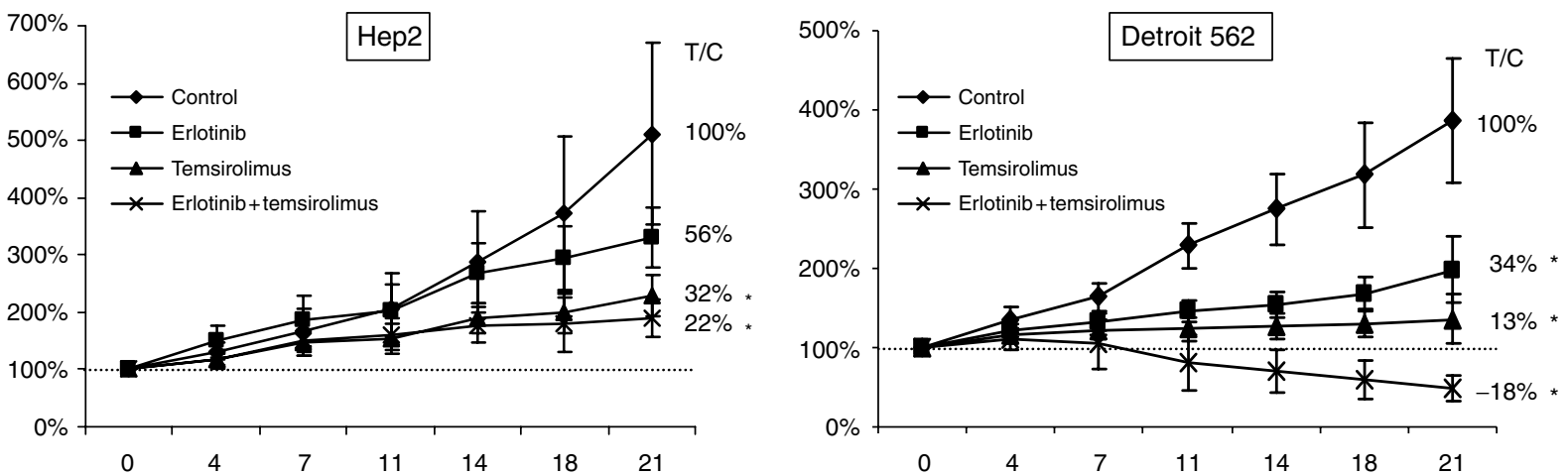

Figure 2 In vivo growth of HEP2 and Detroit 562 cell lines xenografted in nude mice treated with vehicle $(\bullet)$, erlotinib (50 mg kg day ${ }^{-1}$ i.p.; $\left.\mathbf{\square}\right)$, temsirolimus $\left(20 \mathrm{mg} \mathrm{kg} \mathrm{day}^{-1}\right.$ i.p.; $\boldsymbol{\Lambda}$ ) and a combination of both (same doses; $x$ ). The growth of the control group since the day treatment started to day $2 \mathrm{I}$ of the experiment was set as the reference (I00\%). In HEP2 xenograts erlotinib alone did not delay growth significantly, reaching $56 \%$ of the control volume by 21 days. Temsirolimus did decrease growth to $32 \%$, and the combination to erlotinib and temsirolimus induce a growth arrest of $22 \%$ of the control group. However, the differences between the latter two groups were not significant. In Detroit 562, erlotinib alone delayed growth to $34 \%$ of the control volume by 21 days. Temsirolimus slowed growth to 13\%, and the combination actually induced tumour regressions. Error bars represent s.d. $(n=10$ tumours per group). $* P \leqslant 0.05$ compared to control.

cell lines and showed no differences (274 vs 253, $P=0.31)$ (Figure 3A).

On day 7 after treatment, neither Ki67 nor p-MAPK showed a decrement in the HEP2 cell line. Phospho-P70 decreased mildly (15 and 25\%) after single-agent temsirolimus and the combined regimen, respectively. In Detroit 562 tumours, Ki67 and pMAPK only decreased significantly $(>50 \%)$ after combination therapy. Phospho-P70 decreased after single-agent temsirolimus but more dramatically with the combined therapy ( 40 and $60 \%$, respectively) (Figure $3 \mathrm{~B}$ and $\mathrm{C}$ ).

Therefore, FNA on day 7 was able to predict in both groups the activity (or lack of) of the different agents by day 21 . A significant decrease in all three markers was only achieved in the combination arm in the Detroit 562 cell line, and it was the only arm where actual tumour regression was observed. Tumour stability (observed in Detroit 562 treated with temsirolimus, and in HEP2 treated with temsirolimus and the combination) was heralded by modest $(<50 \%)$ decreases in pP70 staining index.

\section{Tumour gene expression analysis}

In each xenografted cell line, 24 tumours (six per treatment arm) were followed by FNA at baseline and on day 7 of treatment for gene expression analysis. The gene expression RT-PCR analysis of the baseline FNA samples showed that HIF- $1 \alpha$ mRNA expression was nonsignificantly lower in HEP2 than in Detroit 562 tumours (7.9 vs 15.1 (arbitrary units), $P=0.064$ ), whereas there was no difference in c-fos (432 vs 708, $P=0.39)$ or EGFR mRNA (6.4 vs 8.5, $P=0.41)$. When days 0 and 7 were compared, HIF- $1 \alpha$ mRNA increased two- and fourfold in HEP2 and Detroit 562 control tumours, respectively. $C$-fos levels increased twofold in Detroit 562 control tumours, whereas no significant change was documented in c-fos in HEP2, or in EGFR mRNA levels in either of the two models. In HEP2 tumours, HIF- $1 \alpha$ increased after treatment with erlotinib, did not change with temsirolimus and decreased three-fold in the combined arm. In Detroit 562 , HIF- $1 \alpha$ did not change with any single agent, but decreased eight-fold in the combined arm. As previously described in similar models (Jimeno et al, 2006), c-fos levels were not suppressed by erlotinib in either HEP2 or Detroit 562 xenografts (data not shown). Epidermal growth factor receptor mRNA levels increased in response to erlotinib in the resistant HEP2, whereas did not change in the intermediately sensitive Detroit 562 , in line with previous reports (data not shown) (Jimeno et al, 2005).

\section{Angiogenesis evaluation}

Tumours were blindly examined for vessels by CD31 staining. The standard MVD showed no significant changes between treatment groups in HEP2 (Figure 4A). In Detroit 562 there was a higher density of vessels than in HEP2. Single-agent erlotinib or temsirolimus administration failed to induce any significant changes in MVD. However the tumours treated with the combination presented a trend towards lower MVD count compared with control tumours (24 vs 43 vessels/HPF, respectively; $P=0.068$ ). Then we implemented a novel scoring system that accounted for the pattern of microvessel invasion in the tumours. In HEP2 there were again no significant differences between treatment groups, as depicted in Figure 4B and C. All Detroit 562 tumours assessed presented vessels in the periphery/ pesudocapsule of the tumour; however, the intratumor growth varied significantly between treatment groups. Most of the control tumours had widespread vessels reaching the centre of the tumour. However, the temsirolimus but more importantly the erlotinib plus temsirolimus group had a decrement in vessel intratumor growth. Half of the tumours in the latter group showed no evidence of vessel budding from the capsule inside the tumour, and none of the tumours treated with the combination had vessels reaching the centre.

\section{DISCUSSION}

There is increasing interest in combining signalling inhibitors in cancer treatment. This study explored if combining treatment with an EGFR and an mTOR inhibitor in an SCC model with intermediate susceptibility to EGFR inhibition. The results show that an mTOR inhibitor alone was superior to an EGFR inhibitor in this model. In addition, the combined treatment was clearly superior in one cell line but not the other with a profound tumour regression elicited in the Detroit 562 line. Fine-needle aspiration aspirate tumour biopsies performed on day 7 after treatment showed greater pathway and angiogenesis modulation in the combined treatment that accurately predicted the tumour growth inhibition observed on day 21 .

The analysis of pathway activation showed an upstream activation in Akt induced by mTOR inhibition in the EGFRresistant cell line; other groups have previously described this phenomenon (O'Reilly et al, 2006). Those authors suggest that cells with constitutive activation of mTOR have a feedback downregulation of receptor TK signalling leading to Akt inactivation. 
Reversal of this feedback loop by mTOR induces Akt activation. In a separate work, two transcripts mediating mTOR expression were identified, cyclin D1 and c-myc, which exhibited differential expression in an Akt-dependent manner: high levels of activated Akt resulted in rapamycin-induced downregulation of expression, whereas low levels resulted in upregulation of expression (Gera et al, 2004). The current report is concordant with the notion that activation of Akt upon mTOR inhibition is a mechanism of
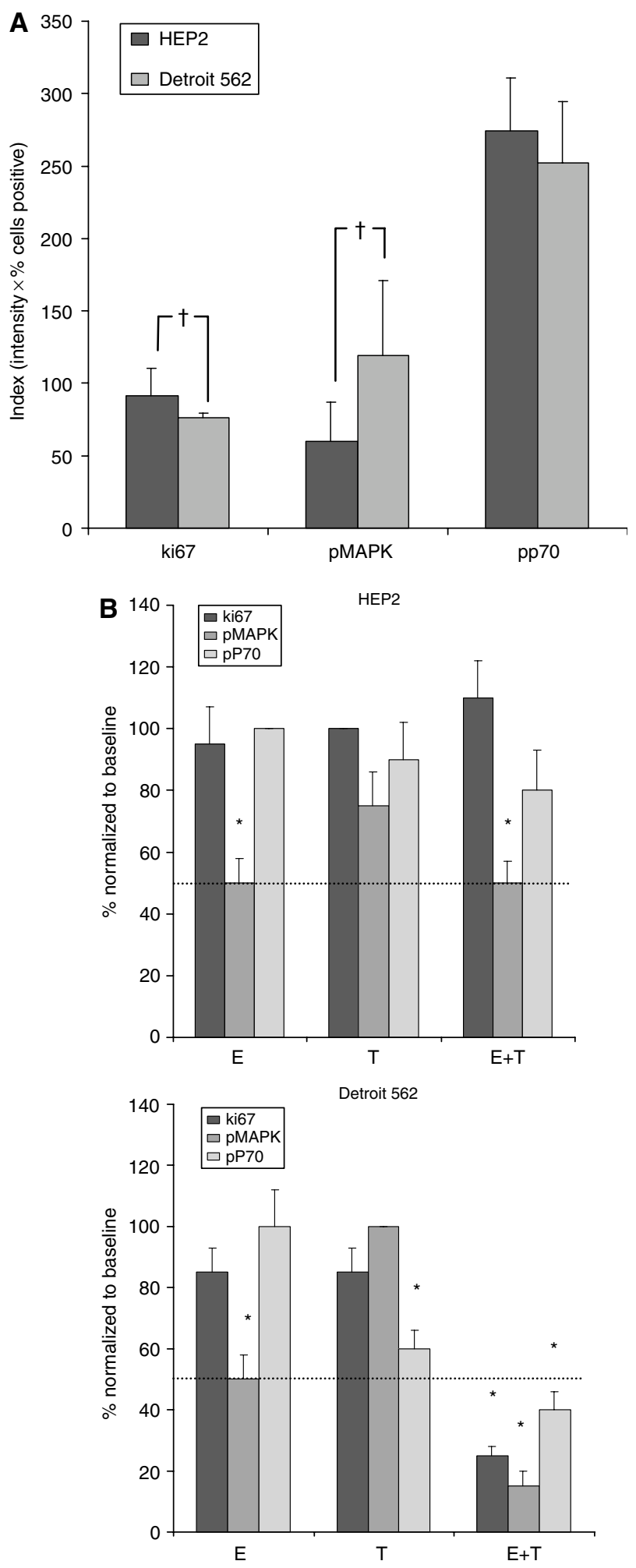

resistance to these agents as the HEP2 cell line in which this phenomenon was observed was clearly resistant. As expected, the two agents combined blunted the activation of Akt induced by temsirolimus. Interestingly, in the cell line where erlotinib was capable of abrogating Akt activity (Detroit 562), an at least additive effect was observed with dual treatment; on the other hand, in HEP2 where erlotinib had no effect on Akt the combined treatment was equally effective as the best single agent. It can be hypothesised that the ability of erlotinib to decrease the level of activation of Akt is important for antitumor effect to occur.

A relevant aspect of this work is the demonstration of the value of FNA-based pharmacodynamic assessment for the prediction of efficacy. Fine-needle aspiration early in therapy was able to predict in both groups the activity (or lack thereof) of the different agents after a conventional treatment cycle; a significant decrease in all three markers was only achieved in the sole treatment arm where actual tumour regression was observed. Inhibition of pMAPK was consistently seen in all four erlotinib-treated groups, but did not correlate with growth inhibitory effect. Globally, this may indicate that the sole assessment of a proximal marker may be inefficient to prognosticate outcome, and combinations of several proximal and distal markers may render better prediction results. In this work we examined two pathway-specific proximal markers (phosphoMAPK and phospho-P70) and a nonspecific distal marker (Ki67) in order to comprehensively assess drug effect. We have previously reported that proximal target inhibition is necessary but not sufficient to achieve antitumor effect (Jimeno et al, 2005, 2006). Interestingly, pathway assessment in vivo correlated better with ultimate treatment efficacy than in vitro signalling. In Detroit 562, the IHC changes were similar to those observed in the cell line Western blots. However, in HEP2 the signalling inhibition was less evident in vivo than in vitro, as little P70 inhibition was documented in the tumours. This may be reflective of a more complex environment, with multiple growth factors and stromal interactions that are not present in vitro. It is important though to bear in mind that none of these techniques is fully quantitative and both have a degree of subjectivity. A combination of three IHC markers was found to be an accurate early predictor of such antitumor activity. This may have implications in targeted therapy development, as new strategies consisting in sequential tumour sampling can be implemented to predict earlier which patients may obtain a benefit, and whom may need additional therapies to control their tumours. This is particularly relevant in SCC of the head and neck or cervix, where biopsies of the primary tumour require invasive procedures not free of potentially severe complications. On the other hand, FNA-based acquisition of tumour material from nodal origin is feasible and safe, as is usually coupled to ultrasound guidance and the quality and accuracy of the procedure can be tested on site by cytopathology personnel.

It is known that mTOR inhibitors work, at least in part, by decreasing angiogenesis (Bruns et al, 2004; Stephan et al, 2004). Although the efficacy of mTOR inhibition and an antiangiogenic

Figure 3 (A) The baseline levels of tumour Ki67, pMAPK and pP70 were averaged and compared with Student's t-test. Proliferation as assessed by Ki67 was higher in HEP2 and PMAPK was higher in Detroit 562. (B and C) Analysis and microphotographs of FNA cores. Variation in Ki67, pMAPK and pP70 levels were assessed by means of sequential FNAs of tumours at baseline and after 7 days of therapy. Each day 7 value was normalised in percentage to its baseline value. Values in the $Y$ axis represent percentage change compared to baseline. Phospho mitogen-activated protein kinase decreased in all four arms treated with erlotinib; in Detroit 562 the $I H C$ changes were similar to those observed in the Figure I Western blots. However, in HEP2 the signalling inhibition was less evident in vivo than in vitro, as little pP70 inhibition was documented. Indeed, pP70 only decreased significantly in Detroit 562 tumours. The only group where a significant ( $\geqslant 50 \%$; cutoff arbitrarily selected) decrease in all three markers was observed corresponded to the combination arm of Detroit 562 $\dagger P \leqslant 0.05$. * $P \leqslant 0.05$ compared to baseline control. 
C

HEP2

Ki67

Detroit 562

pMAPK

Detroit 562

pP70

Detroit 562

HEP2
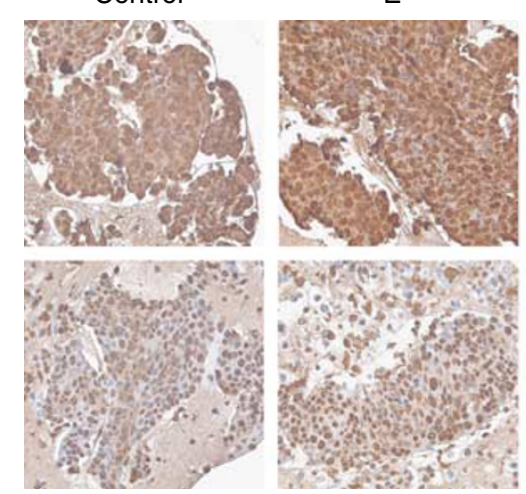

Control
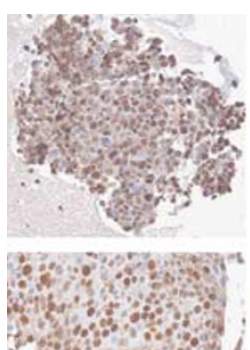

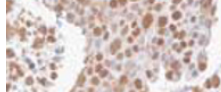

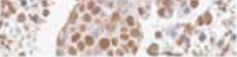
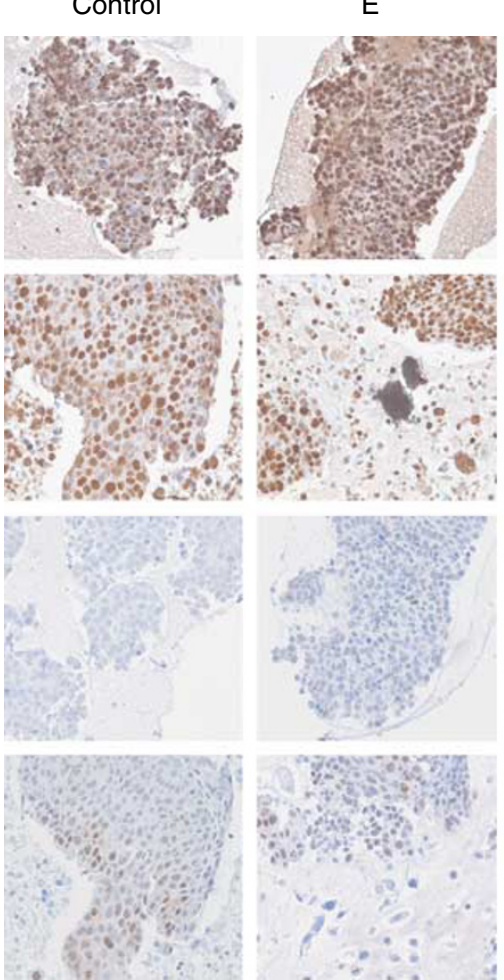
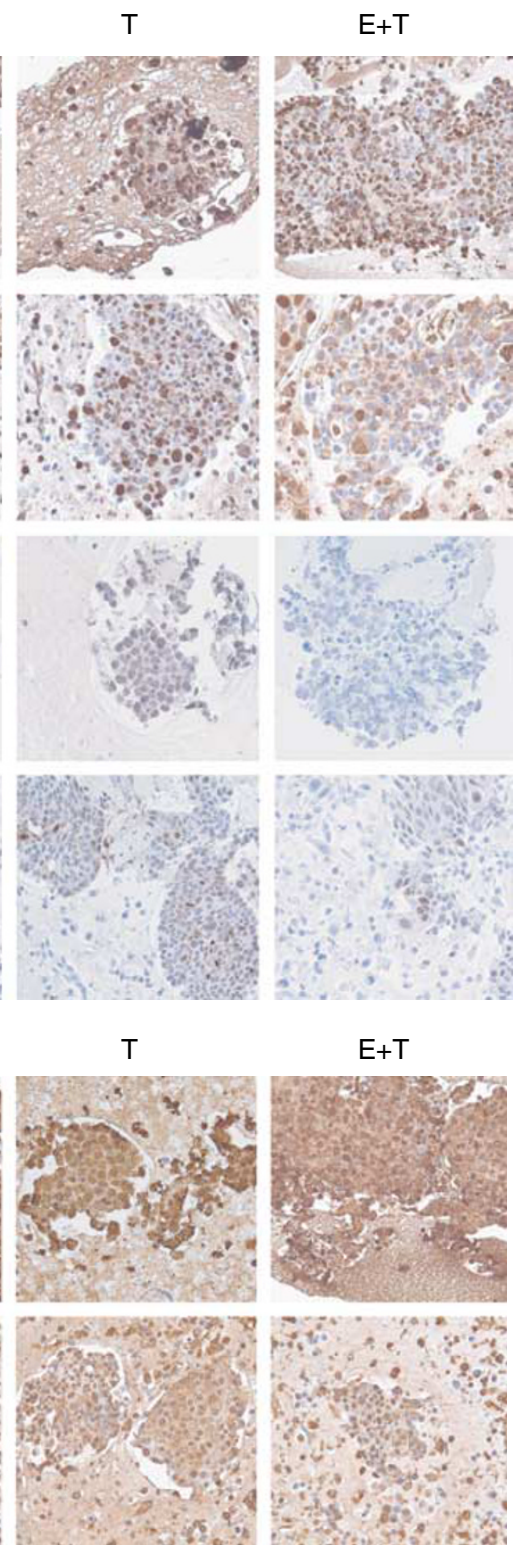

$E+T$

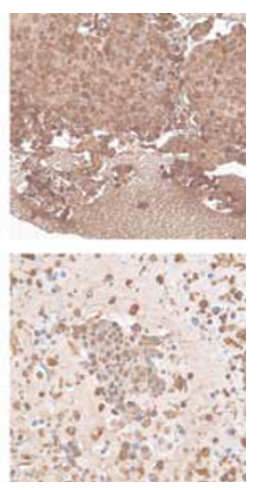

Figure 3 (C) Continued.

effect have already been reported in similar SCC models (Amornphimoltham et al, 2005), the authors presented no direct link between these two phenomena, and hypothesised that the decrease in the vascular network may represent a direct consequence of the rapid reduction in the tumour size rather than being the cause of such antitumor effect. In the presented work, an upregulation of HIF- $1 \alpha$ in tumours over time as their size increased was observed, that may be secondary to an increased hypoxia with tumour growth. In our model only dual EGFR and mTOR inhibition was capable of preventing HIF- $1 \alpha$ upregulation in vivo. It has been suggested that HIF- $1 \alpha$ is modulated by two afferent arms, one from the EGFR pathway and other from the PI3K/Akt pathway (Phillips et al, 2005), and we hypothesise that only a simultaneous inhibition of both afferent arms was able to abrogate HIF- $1 \alpha$. However, this observation needs to be cautiously interpreted, as although HIF- $1 \alpha$ downregulation was seen in both combined arms, only in Detroit 562 there was decreased angiogenesis. Detroit 562 tumours expressed more HIF- $1 \alpha$ at baseline, and also had a higher degree of increase over time with tumour growth, as seen by the 400 vs $200 \%$ increases over 7 days in
Detroit $562 v s$ HEP2 tumours. This may indicate more dependence on HIF- $1 \alpha$ to sustain vessels growth, and thus may explain the higher sensitivity to mTOR inhibition. Currently we do not have an explanation for the induction of HIF- $1 \alpha$ in the EGFR-resistant cell line treated with erlotinib. Overexpression of proangiogenic factors has been shown to be an acquired mechanism of resistance of A431 squamous cell line to anti-EGFR therapies (Viloria-Petit et al, 2001; Viloria-Petit and Kerbel, 2004).

Per standard microvessel assessment, only the combination of erlotinib and temsirolimus in Detroit 562 induced inhibition of angiogenesis. Vessel growth followed a centripetal pattern from the tumour capsule, likely by recruiting murine stromal cellular components, and thus was an adequate model to test the impact of angiogenesis inhibition. We applied a novel method that examines the tumours in their entirety and that allowed us to examine this external invasive pattern of vessel formation; this novel approach correlated well with standard MVD. After breaking the blind of the analysed samples, it was found that temsirolimus, both alone but to a greater extent in combination with erlotinib, was able to inhibit capillary ingrowth in Detroit 562 xenografts but 


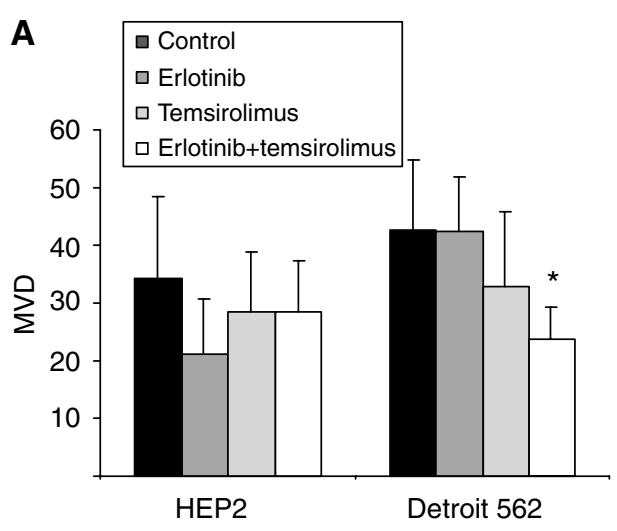

C

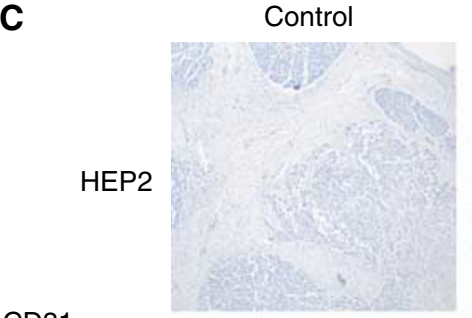

CD31

Detroit 562

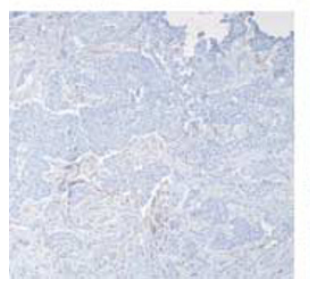

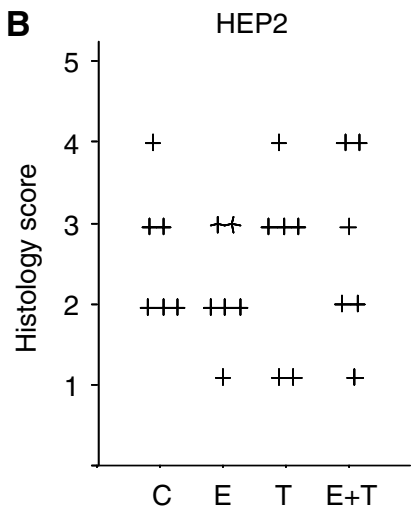

E
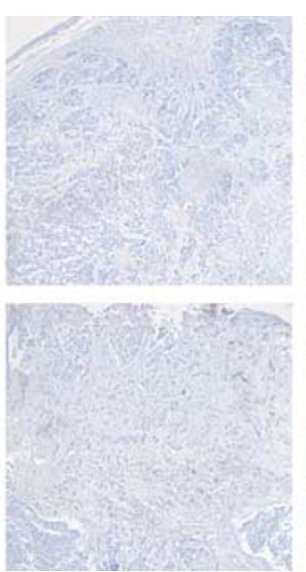

$\mathrm{T}$
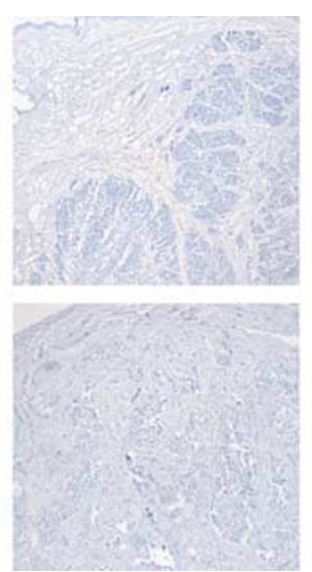

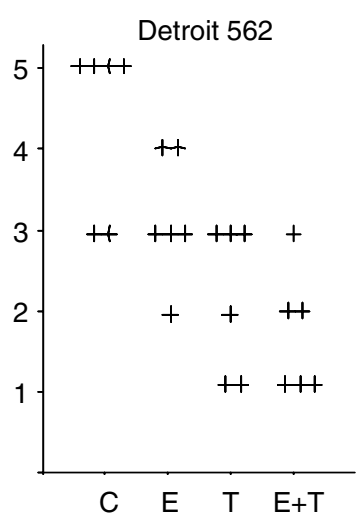

$E+T$

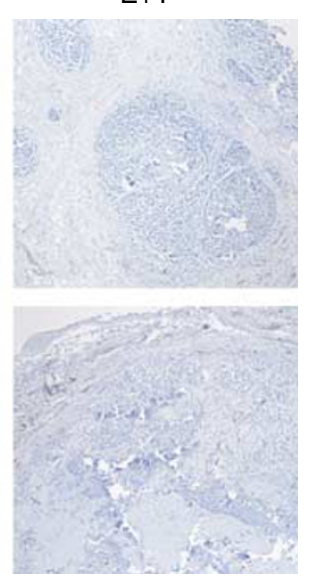

Figure 4 (A) MVD was blindly scored on four areas in each of three tumours per group. In HEP2 no significant changes were seen. In Detroit 562 , the tumours treated with the combination had 24 vs 43 vessels on average $(P=0.068)$. (B) Vessel in-growth assessment. Tumours were blindly scored $I-5$ depending on the extent of vessel invasion inside the tumour. Each individual tumour is represented by a ' + ' symbol. In HEP2 there were no significant differences between treatment groups. In Detroit 562 tumours, a higher density of vessels was evidenced than in HEP2. Most of the control tumours in both cell lines had vessels reaching the centre of the tumour. In Detroit 562 the temsirolimus and to a greater extent the combined arm had a great decrement in vessel intratumor growth. Half of the tumours in the latter group showed no evidence of vessel invasion from the capsule inside the tumour, and none had vessels reaching the centre. $(\mathbf{C})$ Representative images from the above groups. Error bars indicate s.d. C: control; E: erlotinib; T: temsirolimus. * $P \leqslant 0.05$ compared to control.

not in HEP2 tumours. The fact that despite this absence of antiangiogenic effect HEP2 tumours treated with temsirolimus had a significant growth decrement suggests that mTOR inhibitors work by multiple mechanisms as previously described. It also implies that the antitumor effect documented in Detroit 562 xenografts may also be related to mechanisms other than angiogenesis alone.

Cancer is the result of numerous alterations key cellular pathways and genes (Sjoblom et al, 2006), and blocking several of these may be necessary to achieve an antitumor effect. A reflection of this is the success of relatively nonspecific agents such as sorafenib or of agents that interfere at multiple levels such as bortezomib. Some authors advocate the development of 'magic shotguns' rather than 'magic bullets' as a more realistic and potentially successful approach to tackle a disease of such complexity as cancer (Mencher and Wang, 2005). Combining targeted agents is another rational strategy capable to induce tumour regressions in tumour models, as previously reported (Jimeno et al, 2005).

\section{REFERENCES}

Amador ML, Oppenheimer D, Perea S, Maitra A, Cusati G, Iacobuzio-Donahue

C, Baker SD, Ashfaq R, Takimoto C, Forastiere A, Hidalgo M (2004) An
In summary, mTOR inhibitors showed antitumor activity in EGFR-resistant SCC cell lines. This effect was in part mediated by a decrease in angiogenesis. The combination with an EGFR inhibitor induced an additive effect in one of the models. Assessing pharmacodynamic markers by FNA-mediated biopsies early in therapy was predictive of outcome, showing that simultaneously inhibiting several pathways was required to induce tumour regression. This work represents state-of-the-art targeted therapy considering the recent approval of EGFR inhibitors in the corresponding patient population, and addresses the unmet need of primary or acquired resistance to such therapies.

\section{ACKNOWLEDGEMENTS}

This work was supported in part by P50 CA58184-09 SPORE in Head and Neck Cancer and from Instituto de Salud Carlos III (BF03/00631). 
Amornphimoltham P, Patel V, Sodhi A, Nikitakis NG, Sauk JJ, Sausville EA, Molinolo AA, Gutkind JS (2005) Mammalian target of rapamycin, a molecular target in squamous cell carcinomas of the head and neck. Cancer Res 65: 9953-9961

Amornphimoltham P, Sriuranpong V, Patel V, Benavides F, Conti CJ, Sauk J, Sausville EA, Molinolo AA, Gutkind JS (2004) Persistent activation of the Akt pathway in head and neck squamous cell carcinoma: a potential target for UCN-01. Clin Cancer Res 10: 4029-4037

Bianco R, Shin I, Ritter CA, Yakes FM, Basso A, Rosen N, Tsurutani J, Dennis PA, Mills GB, Arteaga CL (2003) Loss of PTEN/MMAC1/TEP in EGF receptor-expressing tumor cells counteracts the antitumor action of EGFR tyrosine kinase inhibitors. Oncogene 22: 2812-2822

Bonner JA, Harari PM, Giralt J, Azarnia N, Shin DM, Cohen RB, Jones CU, Sur R, Raben D, Jassem J, Ove R, Kies MS, Baselga J, Youssoufian H, Amellal N, Rowinsky EK, Ang KK (2006) Radiotherapy plus cetuximab for squamous-cell carcinoma of the head and neck. N Engl J Med 354: $567-578$

Bruns CJ, Koehl GE, Guba M, Yezhelyev M, Steinbauer M, Seeliger H, Schwend A, Hoehn A, Jauch KW, Geissler EK (2004) Rapamycin-induced endothelial cell death and tumor vessel thrombosis potentiate cytotoxic therapy against pancreatic cancer. Clin Cancer Res 10: 2109-2119

DeGraffenried LA, Fulcher L, Friedrichs WE, Grunwald V, Ray RB, Hidalgo M (2004) Reduced PTEN expression in breast cancer cells confers susceptibility to inhibitors of the PI3 kinase/Akt pathway. Ann Oncol 15: $1510-1516$

Gera JF, Mellinghoff IK, Shi Y, Rettig MB, Tran C, Hsu JH, Sawyers CL, Lichtenstein AK (2004) AKT activity determines sensitivity to mammalian target of rapamycin (mTOR) inhibitors by regulating cyclin D1 and c-myc expression. J Biol Chem 279: 2737-2746

Huang S, Armstrong EA, Benavente S, Chinnaiyan P, Harari PM (2004) Dual-agent molecular targeting of the epidermal growth factor receptor (EGFR): combining anti-EGFR antibody with tyrosine kinase inhibitor. Cancer Res 64: $5355-5362$

Jemal A, Siegel R, Ward E, Murray T, Xu J, Smigal C, Thun MJ (2006) Cancer statistics, 2006. CA Cancer I Clin 56: 106-130

Jimeno A, Kulesza P, Kincaid E, Bouaroud N, Chan A, Forastiere A, Brahmer J, Clark DP, Hidalgo M (2006) C-fos assessment as a marker of anti-epidermal growth factor receptor effect. Cancer Res 66: $2385-2390$

Jimeno A, Rubio-Viqueira B, Amador ML, Oppenheimer D, Bouraoud N, Kulesza P, Sebastiani V, Maitra A, Hidalgo M (2005) Epidermal growth factor receptor dynamics influences response to epidermal growth factor receptor targeted agents. Cancer Res 65: 3003-3010

Kirby AM, A'Hern RP, D'Ambrosio C, Tanay M, Syrigos KN, Rogers SJ, Box C, Eccles SA, Nutting CM, Harrington KJ (2006) Gefitinib (ZD1839, Iressa) as palliative treatment in recurrent or metastatic head and neck cancer. Br J Cancer 94: 631-636
Matar P, Rojo F, Cassia R, Moreno-Bueno G, Di Cosimo S, Tabernero J, Guzman M, Rodriguez S, Arribas J, Palacios J, Baselga J (2004) Combined epidermal growth factor receptor targeting with the tyrosine kinase inhibitor gefitinib (ZD1839) and the monoclonal antibody cetuximab (IMC-C225): superiority over single-agent receptor targeting. Clin Cancer Res 10: 6487-6501

Mencher SK, Wang LG (2005) Promiscuous drugs compared to selective drugs (promiscuity can be a virtue). BMC Clin Pharmacol 5: 3

Neshat MS, Mellinghoff IK, Tran C, Stiles B, Thomas G, Petersen R, Frost P, Gibbons JJ, Wu H, Sawyers CL (2001) Enhanced sensitivity of PTENdeficient tumors to inhibition of FRAP/mTOR. Proc Natl Acad Sci USA 98: $10314-10319$

O'Reilly KE, Rojo F, She QB, Solit D, Mills GB, Smith D, Lane H, Hofmann F, Hicklin DJ, Ludwig DL, Baselga J, Rosen N (2006) mTOR inhibition induces upstream receptor tyrosine kinase signaling and activates Akt. Cancer Res 66: $1500-1508$

Phillips RJ, Mestas J, Gharaee-Kermani M, Burdick MD, Sica A, Belperio JA, Keane MP, Strieter RM (2005) Epidermal growth factor and hypoxiainduced expression of CXC chemokine receptor 4 on non-small cell lung cancer cells is regulated by the phosphatidylinositol 3-kinase/PTEN/ $\mathrm{AKT} / \mathrm{mammalian}$ target of rapamycin signaling pathway and activation of hypoxia inducible factor-1alpha. J Biol Chem 280: 22473-22481

Sjoblom T, Jones S, Wood LD, Parsons DW, Lin J, Barber T, Mandelker D, Leary RJ, Ptak J, Silliman N, Szabo S, Buckhaults P, Farrell C, Meeh P, Markowitz SD, Willis J, Dawson D, Willson JK, Gazdar AF, Hartigan J, Wu L, Liu C, Parmigiani G, Park BH, Bachman KE, Papadopoulos N, Vogelstein B, Kinzler KW, Velculescu VE (2006) The consensus coding sequences of human breast and colorectal cancers. Science 314: $268-274$

Soulieres D, Senzer NN, Vokes EE, Hidalgo M, Agarwala SS, Siu LL (2004) Multicenter phase II study of erlotinib, an oral epidermal growth factor receptor tyrosine kinase inhibitor, in patients with recurrent or metastatic squamous cell cancer of the head and neck. J Clin Oncol 22: $77-85$

Stephan S, Datta K, Wang E, Li J, Brekken RA, Parangi S, Thorpe PE, Mukhopadhyay D (2004) Effect of rapamycin alone and in combination with antiangiogenesis therapy in an orthotopic model of human pancreatic cancer. Clin Cancer Res 10: 6993-7000

Viloria-Petit A, Crombet T, Jothy S, Hicklin D, Bohlen P, Schlaeppi JM, Rak J, Kerbel RS (2001) Acquired resistance to the antitumor effect of epidermal growth factor receptor-blocking antibodies in vivo: a role for altered tumor angiogenesis. Cancer Res 61: 5090-5101

Viloria-Petit AM, Kerbel RS (2004) Acquired resistance to EGFR inhibitors: mechanisms and prevention strategies. Int J Radiat Oncol Biol Phys 58: 914-926

Weidner N, Semple JP, Welch WR, Folkman J (1991) Tumor angiogenesis and metastasis - correlation in invasive breast carcinoma. $N$ Engl J Med 324: $1-8$ 